\title{
Some Key Problems for Robot-Assisted Movement Therapy Research: A Perspective from the University of California at Irvine
}

David J. Reinkensmeyer, Member, IEEE, Jose A. Galvez, Laura Marchal, Eric T. Wolbrecht, James E. Bobrow

\begin{abstract}
The field of robot-assisted movement therapy grew rapidly over the past ten years. In this paper we discuss three problems that the field will likely need to address in order to continue to flourish. These problems are to: 1) define the specific benefits of robotic actuation 2) increase the magnitude of functional benefits of robotic training; and 3 ) identify the mechanisms of motor learning in robot-manipulated environments. We review recent research in our laboratory that is addressing these problems. These projects are identifying motor learning tasks that robotic assistance can enhance, developing non-robotic technology when appropriate, and optimizing the forms of robotic assistance to the motor learning properties of humans.
\end{abstract}

\section{INTRODUCTION}

$\mathrm{T}$ HE field of robotic movement therapy began in earnest in 1997 with the publication of the results from the first clinical study of MIT-MANUS, a planar, two-joint robot that can assist patients in moving their arms across a tabletop [1]. This study found that sub-acute stroke patients who received additional movement training with MIT-MANUS recovered significantly better than patients who received only standard rehabilitation therapy. Since that time, tens of research groups and several companies have begun developing robotic movement therapy devices for the arm, hand, and legs, and major public and private funding agencies in the U.S., including NIH, NIDRR, NIST ATP, and the Christopher Reeve Foundation are providing millions of US dollars of funding to support this work. Subsequent clinical studies of robotic movement training have also been positive, to the extent that a recent systematic review of robotic therapy concluded that robotic therapy is well suited for improving proximal upper extremity strength, and might promote motor recovery to a greater extent than traditional therapy [2].

Despite this progress, the field of robotic movement therapy faces serious challenges ahead. We first describe three core problems that we believe the field must address in order to continue to flourish, and then describe several of our own research projects that are aimed at addressing these problems.

Manuscript received February 14, 2007. This work was supported by NIDRR H133E020732, NIDRR H133E020732, NIH N01-HD-3-3352, NCRR M01RR0082, and the Christopher Reeve Foundation.

The authors are with the Department of Mechanical and Aerospace Engineering, University of California at Irvine, 92617 USA (email correspondence to dreinken@uci.edu)

\section{Key Problems For RoBotic MOVEMENT THERAPY}

\section{A. It has not been demonstrated yet that robots are necessary to achieve observed therapeutic benefits}

It is has been established that robotic movement therapy can be beneficial for reducing upper [2] and lower extremity movement impairment $[3,4]$ following stroke and spinal cord injury (SCI). However, control groups have often not been chosen to identify the specific benefit of robotic devices [5]. A robot is a robot by virtue of its capacity to move in response to commands; such movement requires actuators. What is unknown is whether actuators (and thus robots when they actuate patient's limbs) provide therapeutic benefits beyond those associated with the patient's effort to move in and of itself [5]. Put another way, could patients gain comparable therapeutic benefits if the actuators were removed, the joints of the robot were free to move, the same software were used for patient feedback, and the same exercise duration were used? In at least one study of the upper extremity after stroke, the answer was yes, suggested that it is the effort by the patient rather than the assistance by the robot that primarily drives neuroplasticity [6]

\section{B. Therapeutic benefits of robotic therapy are small.}

Even if actuators are beneficial, the therapeutic benefits of robotic therapy that have been measured so far are small. For the upper extremity, one way to gauge benefit is a clinical scale called the Fugl-Meyer score. The Fugl-Meyer score is based on assigning a score of 0 (no movement), 1 , (abnormal movement), or 2 (normal movement) to 33 desired movements, such as raising the arm while keeping the elbow extended, for a maximum score of 66 . Gains in Fugl-Meyer score due to robotic therapy are typically in the range of 2-6 points [7]. The Fugl-Meyer score measures reductions in impairment; increases in functional measures are typically small or non-significant. For robotic locomotor training, a common outcome measure is the walking speed of a 10-meter walk. Typical gains in walking speed attributable to robotic therapy after stroke are around 0.1 $\mathrm{m} / \mathrm{sec}[3,4]$. Thus, while impairment reduction offered by robotic therapy is meaningful to patients, patients must practice intensively - several hours a week for many weeks, in order to regain incremental amounts of movement ability.

A notable exception to these small gains comes from the work of Hesse with the upper extremity [8]. Sub-acute stroke patients who trained with the bi-manu-trac device, which moves the wrist in supination/pronation or flexion/extension, gained 15 additional points on the FuglMeyer score, compared to those who got FES training of the wrist muscles. Understanding why patients benefited more from this device and protocol is an important question for future research. 


\section{We do not understand how motor leaming during neuro-rehabilitation works at a level of detail sufficient to dictate robotic therapy device design.}

Perhaps the most fundamental problem that robotic movement therapy must address to continue to make progress, however, is that we understand very little about how the sensory motor system re-organizes itself in response to robotic movement training. Research on the response of the motor system to training has made several important conceptual breakthroughs in the past twenty years, including the observation that the motor cortex re-organizes the representations of the upper extremity following both injury and training $[9,10]$, the observation that the isolated mammalian spinal cord can learn motor tasks including walking [11], and the observation that the motor system continually forms and updates internal models of the dynamics of its limbs [12]. However, our understanding of the behavioral mechanisms that prompt such re-organization is still basic: we know that repetition, with active engagement by the participant, promotes re-organization $[9$, 10]. We also know that kinematic error drives motor adaptation [13-15]. But the precise ways that mental engagement, repetition, and kinematic error translate into a pattern of recovery is not well defined for rehabilitation.

This gap in knowledge is a problem because engineers cannot design robotic movement training algorithms from first principles. To draw an analogy, if one understands kinematics thoroughly, one can synthesize a mechanism to move through a desired set of paths. No such theoretical basis exists for robotic movement training: we do not understand motor recovery in sufficient detail to synthesize optimal training mechanisms or algorithms.

\section{RESEARCH UNDERWAY AT U.C. IRVINE TO ADDRESS THESE ISSUES}

We are attempting to address these issues with work that is aimed at identifying motor learning tasks that robotic assistance can enhance, developing non-robotic technology when appropriate, and optimizing the forms of robotic assistance to the motor learning characteristics of humans.

\section{A. Research Direction: Identify motor leaming tasks that can be enhanced with robotic assistance.}

There is a joke that if you consult an orthopedic surgeon about what to do about a stuffy nose, he will suggest cutting off your nose. In the same way, we can poke fun of ourselves as roboticists by saying if a rehabilitation clinician consults us about how to help someone learn a motor task during rehabilitation, we will suggest a multiple degrees-offreedom exoskeleton that is capable of mechanically forcing the person to do the task. Clearly, we need better guidelines for what rehabilitation tasks can actually be enhanced by robotic assistance.

The answer to this question will be informed by results in the field of "guidance" in the motor learning literature. Guidance refers to mechanically assisting a person in performing a motor task in order to help him or her learn it [16], which is the therapeutic paradigm adopted by most robotic therapy devices (although, see [17] for erroramplifying approaches). Only a few studies of guidance have been published, focusing mainly on hand positioning tasks. These studies have generally not found significant benefits of guidance for motor learning (e.g. [18-20].

We recently revisited the issue of whether guidance can help with motor learning, but for a more dynamic task than has typically been studied in guidance studies, and one that seemed to us well suited to guidance - learning to drive a vehicle. We developed a control algorithm for a force feedback steering wheel that robotically assists in steering a virtual power wheelchair through a virtual room (Fig. 1). The particular application we have in mind is trying to partially automate wheelchair's driver's training for very young children with cerebral palsy. In other words, we want to give children a way to practice driving a wheelchair without always having a rehabilitation therapist walk next to them and provide hand-over-hand assistance.

We developed a method for systematically reducing (or "fading") the guidance provided by the steering wheel based on real-time measurement of steering errors. Specifically, we adapted the stiffness of the robotic guidance $K$ at the $i^{\text {th }}$ time step using an error-based learning law with forgetting:

$$
\mathrm{K}_{\mathrm{i}+1}=\mathrm{f} \mathrm{K}_{\mathrm{i}}-\mathrm{ge}_{\mathrm{i}}
$$

where $0<\mathrm{f}<1$ is a "forgetting factor" and $\mathrm{g}>0$ is a "learning gain". We used this type of learning law because it tries to reduce the level of robotic guidance when errors are small because of the forgetting factor. Further, it can be shown that such a learning law is used by the human motor system to overcome a predictable disturbance during reaching and walking [15], and further, that it minimizes a cost-function with error and effort terms [21]. The problem of robotic assistance-as-needed can similarly be posed as an optimization problem: minimize kinematic error, but also minimize robotic assistance force [22]. An error-based learning law with forgetting like (1) is one way to achieve this minimization $[22,23]$.

We found that subjects who trained with such "guidanceas-needed" still learned how to steer but with significantly smaller tracking errors. Further, training with guidance-asneeded produced smaller final tracking errors than training without guidance, because subjects apparently learned from the robotic forces better when to initiate turns (Figure 1).

These results are to our knowledge the first evidence that robotic guidance can enhance learning of a motor task, when compared to a non-guided condition. Based on these results, we hypothesize that a domain in which robotic guidance will be useful for rehabilitation is in teaching timing for tasks in which timing is a critical element. Perhaps robotic guidance provides a sensory cue that can be used by the motor system to determine precisely when a motor command should be initiated within an ongoing sensory motor sequence. In contrast, robotic guidance may be less appropriate for demonstrating the required magnitude of the motor command for the movement, since it alters the relationship between muscle force and movement for the task, and thereby obscures the motor command needed to complete the task unassisted. 

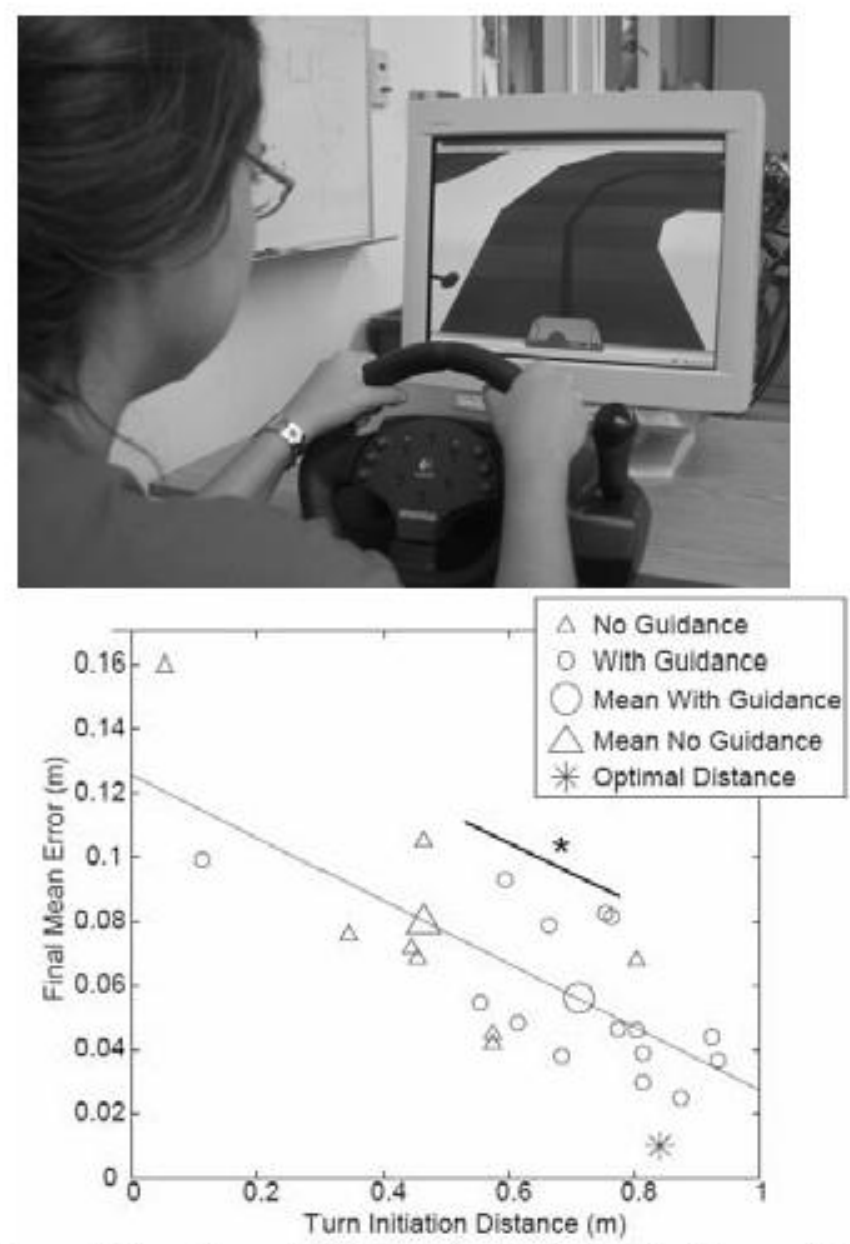

Figure 1: T cp: We used a forcefeedback stering wheel to provide guidance forces that taught uninpaired subjects how to drive a simulated wheelchair that moved at a constant velocity along a track Botbm: After training with an adaptive guidance algprithm, the subjects who received training with robotic guidance ( $(n=15)$ had significantly smaller tracking errors than those who received no guidance $(n=8, p \leq 0.05)$. The final traching error was correlated wilh the turn initiation distance - ies how far ahead of each turn the subjects initiated turns. Subjects who received guidance leamed to initiate turns earlier, closer to the more optimal distance that the robotic steering wheel demons trated. Note that the asterisk denotes the turn initiation distance and tracking error when the steering wheel steered by itself, with an optimized feedback law, without a human operator. The subjects whe trained with guridance (circles) behaved more life the rob ot when guidance was removed.

Taking a step back, what we would like to suggest is that there may be specific types of motor leaming tasks for which robotic guidance can be effective, and others for which it is not helpfil. Identifying the tasks that can benefit from robotic assistance is an important direction for future research. When we have rigorously identified these tasks, we will at the same time have shown that actuators can matter. Further, the functional benefits of robotic therapy for these tasks will likely be more substantial.

\section{B. Research Direction: Develop non-robotic therapy technology when appropriate}

We recently found no significant benefit to robotic assistance for re-training reaching after chronic stroke when compared to an equally-intense, non-assisted training paradigm [6]. This led us to consider how non-robotic devices could be used to enhance motor rehabilitation. Our work here has recently focused on a passive arm orthosis called T-WREX [24], which uses rubber bands to help lift the arm, a grip sensor to sense even small amounts of grip force, and joint sensors to measure arm movement. TWREX stands for Therapy-WREX, and is based on "WREX", a mobile arm support developed for children with am weakness by Dr. Tariq Rahman and colleagues of the A.I. Dupont Hospital for Children [25]

We report in another paper at this conference the details of an ongoing randomized, controlled study of motor training with T-WREX, compared to a matched amount of conventional, self-directed tabletop exercises following chronic stroke. Briefly, the preliminary results from this ongoing study indicate that repetitive motor training with $T$ WREX can reduce motor impairment for chronic stroke survivors with moderate to severe upper extremity hemiparesis. Individuals in the T-WREX $(\mathrm{n}=11)$ and control groups ( $\mathrm{n}=12$ ) demonstrated significant improvements in arm movement ability according to the Fugl-Meyer scale (3.7 and 2.7 points on average, respectively). Subjects in both groups reported nearly significant gains on a functional scale that grades quality and amount of affected arm use in the home setting. There were no significant differences between groups with the current sample size. However, subjects in both training groups strongly preferred the use of T-WREX for therapy, based on a survey given post-training, even after they were given a chance to try both types of therapy.

These results demonstrate that upper extremity movement practice with a non-robotic, exoskeleton can reduce motor impairment, confirming previous pilot study results [24]. Similar results may be possible with the lower extremity [26]. Further, based on these results, we hypothesize that movement practice with robotic or non-robotic devices can enhance patient motivation, as long as the devices provide some sort of interactive feedback. The ability of non-robotic

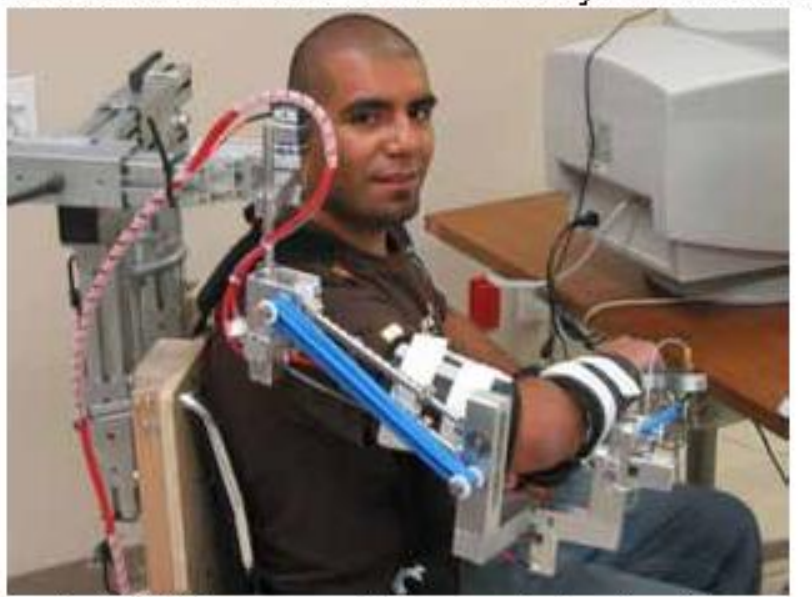

Figure 2: T-WREX - a non-robotic approach to aubmating upper extremity movement training. The orthosis provides gradab le support for the arm against gravity using elastic bands, and measures arm movement and hand grasp as the user interacts with computer simulations of functionalactivities. 
therapy devices to compete with robotic ones in training effectiveness highlights the need to better identify the specific benefits of robotic devices, and to invest effort in non-robotic, motivating technology as well.

\section{Research Direction: Optimize robotic assistance to promote engagement and effort and allow some error}

As described above, we know that effort, engagement, and error matter for motor recovery. For a rehabilitation robot, promoting engagement and effort may be as simple as providing ongoing feedback about the patient's participation. For example, the Lokomat [27] is a gait training device that can be used to force a patient's legs through a stepping trajectory with large impedance. In such a case, the patient can choose to fall asleep in the device and still "walk". However, patients typically don't fall asleep, and it has been shown that the provision of visual feedback that measures participation, such as the size of the contact force against the device, can induce patients to try harder [28]. Thus, when patients are captured in a relatively rigid robot orthosis, it appears that they can voluntarily modulate their effort, and that bio-feedback can further facilitate their effort.

However, walking in a stiff robot prevents kinematic errors that may be helpful for learning. In other words, if a device is less stiff, it has the advantage that variations in motor commands created by the patient will register as variations in the movement pattern of the robot-patient assembly. Thus, compliance maintains the causal relationship between effort and motion, which may be helpful both for motivation and for motor learning.

We have been studying experienced rehabilitation therapists in order to understand how they provide manual assistance for a complex task like body-weight supported gait training [29]. Recent findings suggest that therapists act like position-controlled robots, but only at key moments in the step cycle. Figure 3 shows the estimated limb stiffness of an experienced therapist as she manually assisted at the knee of a severely impaired (ASIA B) person with SCI during the stance and swing phase of walking on a treadmill. The therapist appeared to act like a position controller at the knee at the beginning of both swing and stance, but then acted like a pure force controller with very low stiffness throughout much of stance and swing. This strategy maintained a general kinematic pattern of stepping but allowed some variability (e.g. 5-10\% of the size of the step), rather than enforcing one repeated, ideal kinematic pattern.

How can a therapist move a limb in a general pattern without relying on position control? The likely answer is that they form an internal model of the dynamics of the patient's limbs and use force control. In other words, the therapist senses the movement capability of the patient, using sight and feel, and then adjusts his or her level of assistance force to the patient's need. This allows the therapist to apply an appropriate force without continuously relying on a stiff position control strategy.
A. Experimental set-up and sample regression to identify stiffness
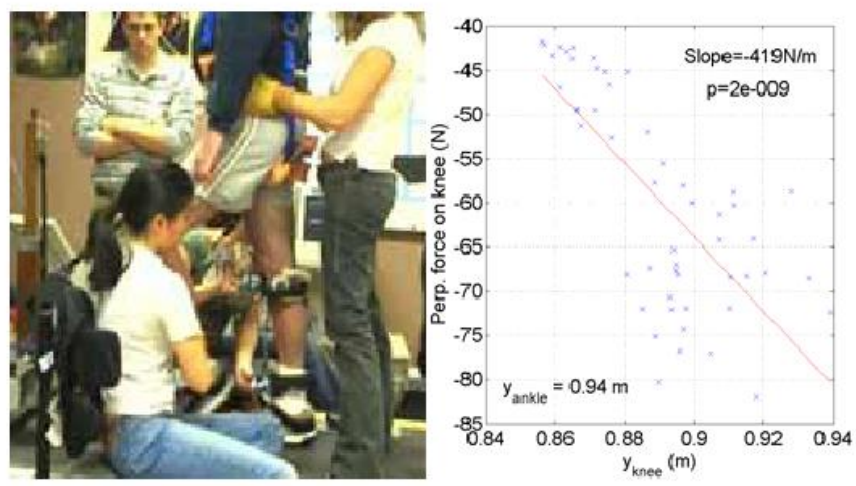

B. Swing phase horizontal stiffness at knee. Heel strike is right.

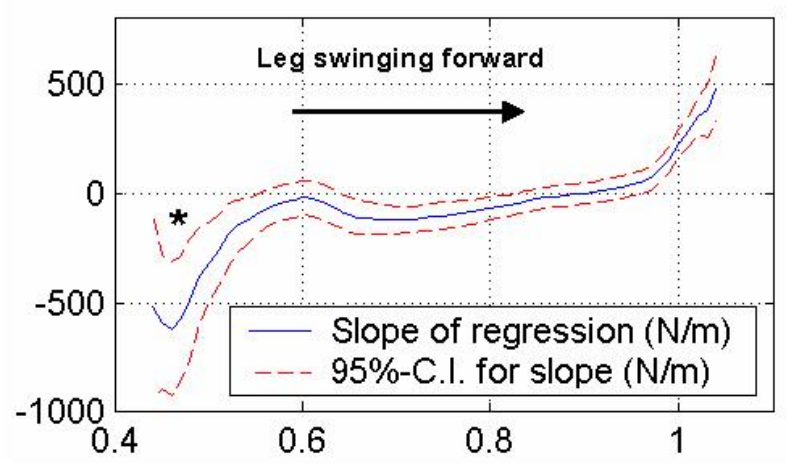

C. Stance phase horizontal stiffness at the knee. Heel strike is right.

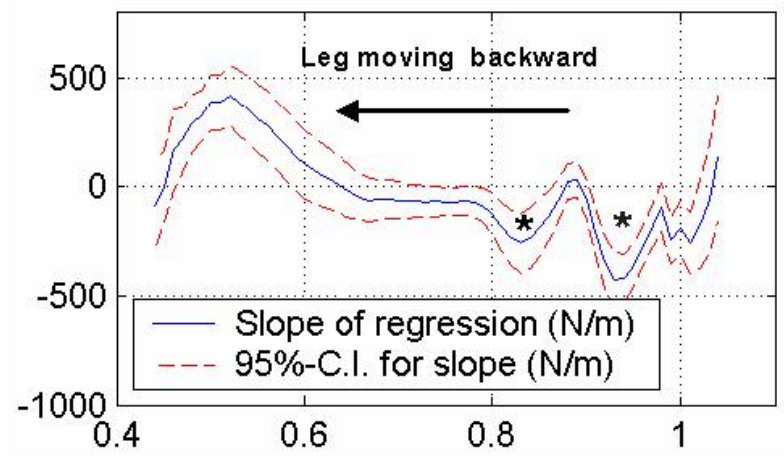

Fig. 3 A) An instrumented orthosis for measuring how therapists control a subject's leg during body weight supported locomotor training on a treadmill. The subject had an SCI and was classified as ASIA B. We used the orthosis to measure the forces and motions applied to the knee and ankle during manual assistance. To identify the stiffness at the knee due to the therapist and patient, we regressed the horizontal force applied to the knee versus the change in knee horiz ontal position, relative to the mean position, at samples along the step cycle from step to step (A - right). B) Identified knee stiffness during swing. The stiffness is plotted versus the position of the ankle during the gait cycle. A negative stiffness corresponds to a stabilizing controller, like a position controller. C) Identified knee stiffness during stance. Note that the therapist acted like a proportional position controller for a short time at the beginning of swing and stance (i.e. negative stiffness is significantly different from zero at the locations denoted by *). The identified stiffness was near zero throughout much of the rest of the step cycle, even though substantial assisting forces (not shown) were applied to the knee. Stiffness became positive (i.e. like an unstable spring) when therapist helps change leg direction at end of swing and end of stance. 
How then can a robot be made to use force control in a similar way to successfully assist an impaired person in moving in a desired pattern, but still allow that person to influence the movement trajectory? One approach is to use established techniques in adaptive control theory to learn the appropriate amounts of force to help move the person's limbs. We recently investigated the use of one standard adaptive controller (an "adaptive sliding controller" [30]) for the task of moving a severely weakened patient's arm between two targets following stroke. Subjects placed their arm in a compliant robotic exoskeleton called Pneu-WREX [31] that allows spatial motion of the hand, and then tried to track a cursor as it moved between two locations at chest level in front of their body. Many stroke patients are incapable of doing this task since it requires holding the hand up in front of the body against gravity. The stiffness of the exoskeleton was set at $70 \mathrm{~N} / \mathrm{m}$, which is at the low end of what one might expect from a therapist's arms [32].

The compliant exoskeleton could not rigidly move the subject's arm through the task, so, as subjects tried to perform this task, we used an adaptive sliding controller to update a model of the forces required to lift the patient's $\mathrm{arm}$. The model was constructed by adjusting the parameters of radial basis functions based on tracking error measurements. We found that this type of adaptive controller successfully learned the assistance forces necessary to move the relaxed arms of patients to targets. (Fig. 4). However, when we asked subjects to begin to move on their own, we found the interesting property that the adaptive controller "refused" to relinquish control to the subject. In addition, if we initialized the adaptive controller to zero force and asked subjects to move between the two targets, the subjects gradually allowed the controller to take over performance of the support component of the task. In effect, it appears that the human motor system took advantage of the opportunity to do less work when connected to a robot with an adoptive controller that had the potential to take over.

The situation may be different from human interaction with a stiff controller, because with a stiff controller, the patient can do anything and the controller will "block" the force immediately, and stay on course because of its rigidity. With a very compliant controller, if the patient changes the force he or she generates, a kinematic error develops, and then both the patient's motor system and the adaptive controller compete to correct this error over a time frame related to the speed at which they update their internal models of the system dynamics. The human motor system appears to allow a standard adaptive controller to win this competition, perhaps because the standard adaptive controller responds quickly, and by allowing it to respond the motor system achieves the desired movement with less effort.

To engage the human motor system, we incorporated a forgetting rate in the adaptive controller that caused the controller to try to reduce its force when kinematic error was small. We had developed some background theory for this sort of modification based on motor adaptation studies with unimpaired subjects [22], as discussed above in the steering study. Remarkably, inclusion of this forgetting rate caused the subjects to exert significantly more effort for supporting their own arms as they tracked the cursor between the two targets, while at the same time still keeping tracking error small (Fig. 4). With such a controller, subjects have a strong subjective impression that they are creating and influencing the ongoing movement, perhaps because their effort does influence the robot path. We are now using the adaptive sliding controller with forgetting for training stroke subjects with the compliant Pneu-WREX orthosis. We plan on applying it to the compliant gait training robot developed in our laboratory as well (PAM/POGO, [33]).
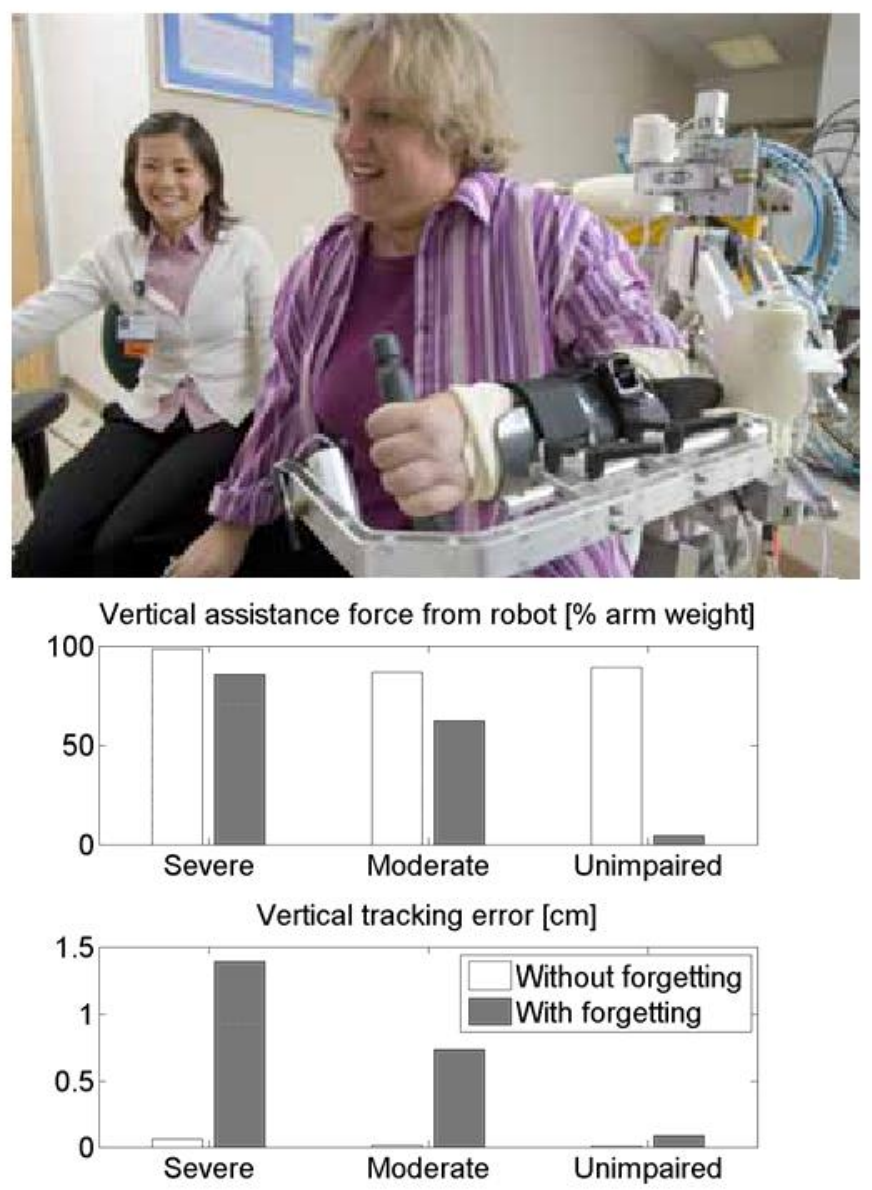

Fig. 4. Top: Person with a stroke receiving movement training with a robotic exoskeleton called Pneu-WREX. Bottom: Mean vertical assistance force provided by Pneu-WREX as a function of patient impairment (severely impaired, Fugl-Meyer $<24, n=4$; moderately impaired, $24<F M<66 \mathrm{n}=4$; unimp aired, $\mathrm{FM}=66, \mathrm{n}=3$ ). The task was to track a cursor that moved between two targets placed in the frontal plane at chest height. With a forgetting rate in the adaptive controller, the robot contributed significant less support force (grey bars versus white bars in top plot, paired t-test across subjects, $\mathrm{p}<$ 0.05 ), indicating that the subjects exerted more force as they performed the task. Tracking error increased with forgetting present, but was still under $1.5 \mathrm{~cm}$ even for severely impaired patients who could not do the task without robot assistance. 


\section{Discussion AND CONCLUSION}

The clinical application of robotic movement therapy will continue to accelerate as researchers validate the use of actuators for therapy, find ways to increase the functional benefits of robotic therapy, and use motor adaptation research to inform their machine designs. As reviewed above, in our own research, we are seeking to identify the categories of tasks for which robotic guidance is effective. One candidate category is tasks for which timing is important, such as steering a vehicle. We are forgoing robotic assistance for simpler non-actuated devices when we can (e.g. T-WREX). We are making robot therapy systems with very low mechanical compliance, like rehabilitation therapists, thereby allowing the patient to directly influence movement kinematics (e.g. Pneu-WREX, PAM/POGO). By necessity, we are then devising adaptive controllers that form real-time models of patient's motor capability in order to move limbs accurately with force control. We are discovering that motor adaptation mechanisms, such as the human tendency for error/effort optimization, impact the way patients interact with such compliant, adaptive, assistive robots. Humans appear to contribute less effort to a rehabilitation task if they are attached to a robot that will adapt and begin to do the task for them. This problem can be addressed by making the robot try to minimize its force also via a forgetting process.

A key goal for future research is to determine whether compliant robot assistance improves the clinical results of robotic movement therapy. We will also try to improve our understanding of the motor adaptation mechanisms that operate during rehabilitation in order to increase the effectiveness of robot-assisted therapy.

\section{ACKNOWLEDGMENT}

We acknowledge the support of Sarah Housman, O.T., Rehabilitation Institute of Chicago, Dr. Susan Harkema, U. Louisville, Dr. Daisuke Aoyagi and Wade Ichinose (Rancho Los Amigos National Rehabilitation Hospital) for research described in this paper.

\section{REFERENCES}

[1]M. L. Aisen, H. I. Krebs, N. Hogan, F. McDowell, and B. Volpe, "The effect of robot-assisted therapy and rehabilitative training on motor recovery following stroke," Arch. Neurol., vol. 54, pp. 443-446, 1997.

[2] G. B. Prange, M. J. A. Jannink, C. G. M. Groothuis, H. J. Hermens, and M. J. IJzerman, "Systematic review of the effect of robot-aided therapy on recovery of the hemiparetic arm after stroke," J. Rehabil. Res. Develop, vol. 43, pp. 171-184, 2006.

[3]C. Werner, S. Von Frankenberg, T. Treig, M. Konrad, and S. Hesse, "Treadmill training with partial body weight support and an electromechanical gait trainer for restoration of gait in subacute stroke patients: a randomized crossover study," Stroke, vol. 33, pp. 2895-901, 2002.

[4]T. Hornby, "Clinical and quantitative evaluation of robotic-assisted treadmill walking to retrain ambulation after spinal cord injury," Topics in Spinal Cord Injury Rehabilitation, vol. 11, pp. 1-17, 2005.

[5]L. Kahn, P. Lum, W. Rymer, and D. Reinkensmeyer, "Robot-assisted movement training for the stroke-impaired arm: Does it matter what the robot does?," Journal of Rehabilitation Research and Development, vol. 43 pp. 619-630 2006.

[6]L. E. Kahn, M. L. Zygman, W. Z. Rymer, and D. J. Reinkensmeyer, "Robot-assisted reaching exercise promotes arm movement recovery in chronic hemiparetic stroke: A randomized controlled pilot study," Journal of Neuroengineering and Neurorehabilitation, vol. 3:12, 2006.
[7]D. Reinkensmeyer, J. Emken, and S. Cramer, "Robotics, motor learning, and neurologic recovery," Annual Review of Biomedical Engineering, vol. 6, pp. 497-525, 2004.

[8]S. Hesse, C. Werner, M. Pohl, S. Rueckriem, J. Mehrholz, and M. L. Lingnau, "Computerized arm training improves the motor control of the severely affected arm after stroke: a single-blinded randomized trial in two centers," Stroke, vol. 36, pp. 1960-6. Epub 2005 Aug 18., 2005.

[9]R. J. Nudo, B. M. Wise, F. SiFuentes, and G. W. Milliken, "Neural substrates for the effects of rehabilitative training on motor recovery after ischemic infarct," Science, vol. 272, pp. 1791-1794, 1996.

[10] J. Liepert, H. Bauder, H. R. Wolfgang, W. H. Miltner, E. Taub, and C. Weiller, "Treatment-induced cortical reorganization after stroke in humans," Stroke, vol. 31, pp. 1210-6, 2000.

[11] V. R. Edgerton, R. D. Leon, S. J. Harkema, J. A. Hodgson, N. London, D. J. Reinkensmeyer, R. R. Roy, R. J. Talmadge, N. J. Tillakaratne, W. Timoszyk, and A. Tobin, "Retraining the injured spinal cord," J Physiol, vol. 533 , pp. 15-22., 2001.

[12] R. Shadmehr and F. A. Mussa-Ivaldi, "Adaptive representation of dynamics during learning of a motor task," Journal of Neuroscience, vol. 14, pp. 3208-3224, 1994.

[13] K. A. Thoroughman and R. Shadmehr, "Learning of action through adaptive combination of motor primitives.," Nature, vol. 407, pp. 742-7, 2000.

[14] R. A. Scheidt, J. B. Dingwell, and F. A. Mussa-Ivaldi, "Learning to move amid uncertainty," Journal of Neurophysiology, vol. 86, pp. 971-85, 2001.

[15] J. Emken and D. Reinkensmeyer, "Robot-enhanced motor learning: Accelerating internal model formation during locomotion by transient dynamic amplification," IEEE Transactions on Neural Systems and Rehabilitation Engineering, vol. 99, pp. 1-7, 2005.

[16] R. A. Schmidt, Motor Control and Learning: Human Kinetics Publishers, 1988.

[17] J. L. Patton, M. E. Phillips-Stoykov, M. Stojakovich, and F. A. Mussa-Ivaldi, "Evaluation of robotic training forces that either enhance or reduce error in chronic hemiparetic stroke survivors," Experimental Brain Research, vol. 168, pp. $368-383,2005$.

[18] C. J. Winstein, P. S. Pohl, and R. Lewthwaite, "Effects of physical guidance and

knowledge of results on motor learning: support for the guidance hypothesis," Research Quarterly for Exercise and Sport, vol. 65, pp. 31632, 1994.

[19] D. Feygin, M. Keehner, and F. Tendick, "Haptic Guidance: Experimental Evaluation of a Haptic Training Method for a Perceptual Motor Skill," Proc. 10th Int. Symp. on Haptic Interfaces for Virtual Environment and Teleoperator Systems (Haptics 2002), Orlando, FL, pp. $40-47,2002$.

[20] J. Liu, S. C. Cramer, and R. D.J., "Learning to perform a new movement with robotic assistance: Comparison of haptic guidance and visual demonstration," Journal of Neuroengineering and Rehabilitation, vol. 31 pp. $20,2006$.

[21] D. J. Reinkensmeyer, J. L. Emken, J. Liu, and J. E. Bobrow, "The nervous system appears to minimize a weighted sum of kinematic error, force, and change in force when adapting to viscous environments during reaching and stepping," presented at Advances in Computational Motor Control III Symp., pp. [Online]. Available: http://www.bme.jhu.edu/acmc/, 2004.

[22] J. Emken, R. Benitez, and D. Reinkensmeyer, "Human-robot cooperative movement training: learning a novel sensory motor transformation during walking with robotic assistance-as-needed," Journal of Neuroengineering and Rehabilitation, vol. to appear, 2007.

[23] D. Reinkensmeyer, D. Aoyagi, J. Emken, J. Galvez, W. Ichinose, G. Kerdanyan, S. Maneekobkunwong, K. Minakata, J. Nessler, W. Timoszyk, K. Vallance, R. Weber, J. Wynne, R. Roy, R. d. Leon, J. Bobrow, S. Harkema, and V. Edgerton, "Tools for Understanding and Optimizing Robotic Gait Training," Journal of Rehabilitation Research and Development, vol. In Press, 2006.

[24] R. Sanchez, J. Liu, S. Rao, P. Shah, R. Smith, S. Cramer, J. Bobrow, and D. Reinkensmeyer, "Automating arm movement training following severe stroke: functional exercises with quantitative feedback in a gravityreduced environment," IEEE Transactions on Neural and Rehabilitation Engineering, vol. 14 pp. 378-389, 2006. 
[25] T. Rahman, W. Sample, R. Seliktar, M. Alexander, and M. Scavina, "A body-powered functional upper limb orthosis," Journal of Rehabilitation Research and Development, vol. 37, pp. 675-80, 2000.

[26] S. K. Agrawal and A. Fattah, "Theory and design of an orthotic device for full or partial gravity-balancing of a human leg during motion," IEEE Trans Neural Sys \& Rehab Eng, vol. 12, pp. 157-165, 2004.

[27] G. Colombo, M. Joerg, R. Schreier, and V. Dietz, "Treadmill training of paraplegic patients with a robotic orthosis," Journal of Rehabilitation Research and Development, vol. 37, pp. 693-700, 2000.

[28] R. Riener, L. Lunenburger, S. Jezernik, M. Anderschitz, G. Colombo, and V. Dietz, "Patient-cooperative strategies for robot-aided treadmill training: first experimental results," IEEE Trans Neural Sys \& Rehab Eng, vol. 13, pp. 380-394,, 2005.

[29] J. A. Galvez, G. Kerdanyan, S. Maneekobkunwong, R. Weber, M. Scott, S. J. Harkema, and D. J. Reinkensmeyer, "Measuring human trainers' skill for the design of better robot control algorithms for gait training after spinal cord injury," Proc. 2005 IEEE International Conference on Rehabilitation Robotics, Chicago, Illinois, pp. 231-234, 2005.

[30] J. J. E. Slotine and W. Li, "On the Adaptive Control of Robot Manipulators," The International Journal of Robotics Research, vol. 6, pp. 49, 1987

[31] R. Sanchez, E. Wolbrecht, R. Smith, J. Liu, S. Rao, S. Cramer, T. Rahman, J. Bobrow, and D. Reinkensmeyer, "A pneumatic robot for retraining arm movement after stroke: rationale and mechanical design," Proceedings of the 2005 IEEE International Conference on Rehabilitation Robotics, June 28-July 1, Chicago, Illinois, vol. 500-504, 2005.

[32] E. Burdet, R. Osu, D. W. Franklin, T. E. Milner, and M. Kawato, "The central nervous system stabilizes unstable dynamics by learning optimal impedance," Nature, vol. 414, pp. 446-9., 2001.

[33] D. Aoyagi, W. E. Ichinose, S. J. Harkema, D. J. Reinkensmeyer, and J. E. Bobrow, "An assistive robotic device that can synchronize to the pelvic motion during human gait training," Proc. 2005 IEEE International Conference on Rehabilitation Robotics, Chicago, Illinois, pp. 565-568, 2005 . 